\title{
Finite size effects in the specific heat of glass-formers
}

\author{
L. A. Fernandez*, V. Martín-Mayor* and P. Verrocchio ${ }^{\dagger}$ \\ *Departamento de Física Teórica I, Universidad Complutense, Avenida Complutense, 28040 Madrid, Spain \\ ${ }^{\dagger}$ Dipartimento di Fisica, Università di Trento, via Sommarive 14, 38050 Povo, Trento, Italy
}

\begin{abstract}
We report clear finite size effects in the specific heat and in the relaxation times of a model glass former at temperatures considerably smaller than the Mode Coupling transition. A crucial ingredient to reach this result is a new Monte Carlo algorithm which allows us to reduce the relaxation time by two order of magnitudes. These effects signal the existence of a large correlation length in static quantities.
\end{abstract}

\section{INTRODUCTION}

When compared to experiments, numeric simulations of glass-forming systems present severe limitations. In fact, while the actual physical origin of the phenomenon termed generically as glass transition is still a matter of debate, its investigation is limited by the practical impossibility to reach thermodynamic equilibrium when the relaxation time $\tau$ of a sample glass in a laboratory becomes of order of $10^{2}$ seconds or when the $\tau$ of a model glass in a modern computer becomes of $\sim 10^{-8}$ seconds. Thus the glass temperature $T_{g}$ is conventionally defined in experiments and numeric simulations cannot get very close to it ${ }^{1}$. Furthermore, the statistical accuracy in experiments is fairly larger, because the time-length $\mathscr{L}$ of typical experiments is many decades larger than in simulations. Indeed, statistical errors decrease roughly as $1 / \sqrt{\mathscr{N}_{\text {ind }}}$ where the number of independent configurations $\mathscr{N}_{\text {ind }}$ is given by $\mathscr{N}_{\text {ind }} \sim \mathscr{L} / \tau$. This problem is particularly serious in the study of time correlators, whose peculiar behavior is one of the most clear signature of the glass transition. For every observable, $O$, the normalized time correlator is

$$
C_{O}(t)=\frac{\langle O(0) O(t)\rangle-\langle O\rangle^{2}}{\left\langle O^{2}\right\rangle-\langle O\rangle^{2}} .
$$

The region $C \sim 0$ is very interesting, since it allows to estimate the relaxation time (see below). On the other hand, in that region the relative error from a single measurement grows as $C^{-1}$ hence a very large $\mathscr{N}_{\text {ind }}$ is needed to get some significant signal over the noise. As a consequence, most of previous numeric work was confined to

\footnotetext{
1 e.g. in $\mathrm{SiO}_{2}$ Molecular Dynamics simulation have been performed only down to $T \sim 2200 \mathrm{~K}$ while $T_{g}=1500 \mathrm{~K}$.
}

$C>0.1$, while in experiments one is able to explore far lesser values of $C$.

Despite those limitations, numeric simulations turn out to be quite useful in many respects, mostly in the key issue of identifying the quantity suffering the largest spatial fluctuations at the glass transition. [1, 2, 3, 4, 5] In fact, unlike standard phase transitions the equilibration time of glass-formers (supercooled liquids, polymers, proteins, superconductors, etc.) diverges without dramatic changes in their structural properties [6, 7, 8], challenging the interpretation of the slowing down as a critical phenomenon[9]. A possible way out has been found in the heterogeneous dynamic scenario, which postulate that local time correlators (i.e where $O$ is a local quantity) are correlated over diverging length scales. The possible advantages of numeric simulations with respect of experiment are twofold. First, they are more suitable to investigate local quantities. Second, they allow an easier implementation of Finite Size Scaling techniques, a powerful tool to detect diverging correlation length. [10, 11, 12] New, fast algorithms for numerical simulation are therefore needed in order to to get as close as possible to the experimental regime, while retaining all the advantages provided by numerics.

\section{TRANSLATIONAL INVARIANT QUANTITIES}

In this numeric work, we wish to ask the following question: Are there static observables whose correlation length $\xi$ diverge at the glass transition? (Note that experimental clues are not available). In other words we wonder if a standard second order phase transition is behind the glass transition. We borrow from the dynamic heterogeneity scenario the hypothesis that a diverging cor- 
relation length would show up in four-particle correlation function and focus on translational invariant quantities.

Little attention has been paid so far to such quantities. In fact experiments (e.g. elastic scattering for the spatial correlations and dielectric response for the dynamic behavior) and simulations typically investigate quantities which are not invariant under an uniform displacement of the coordinates, $\vec{r}_{i}=\vec{r}_{i}+\vec{\Delta}$, namely the density fluctuations of generic momentum $\vec{Q}$ :

$$
\rho(\vec{Q})=\frac{\sigma_{0}^{-3}}{N} \sum_{j=1}^{N} \mathrm{e}^{\mathrm{i} \vec{r}_{j} \cdot \vec{Q}},
$$

where $\rho(0)$ is the particle density and it is constant because of mass conservation. Density fluctuations at small $\vec{Q}$ are surely slow modes of the system, their time-correlator decaying exponentially with characteristic time $\tau \sim D^{-1} Q^{-2}$ ( $D$ is the diffusivity). We have no guarantee whatsoever though that they are the only relevant slow modes.

How to expand the space of observables investigated taking into account even quantities which preserve the translational symmetry? For the sake of definiteness we limit to the $N V T$ ensemble and introduce energy density fluctuations:

$$
\rho_{e}(\vec{Q})=\frac{\sigma_{0}^{-3}}{2 N} \sum_{j, k \neq j} \mathrm{e}^{\mathrm{i} \vec{r}_{j} \cdot \vec{Q}_{V}} V_{k j}
$$

where $V_{k j}$ is the interaction energy $V\left(\vec{r}_{k}-\vec{r}_{j}\right)$ between particles $k$ and $j$. Note that $\rho_{e}(0)$ is the non-conserved potential energy density, which we call $e$ (the internal energy is then $\frac{3}{2} k_{\mathrm{B}} T+\langle e\rangle$ ). A general way to obtain translationally invariant observables consists in multiplying densities with wave-vectors $\vec{q}$ and $-\vec{q}$ :

$$
\mathscr{S}(\vec{q})=\rho(\vec{q}) \rho(-\vec{q}), \quad \mathscr{S}_{e}(\vec{q})=\rho_{e}(\vec{q}) \rho_{e}(-\vec{q}) .
$$

We see then that the correlation functions of these translational invariant quantities involve the positions of four particles. Moreover the mean value of $\mathscr{S}(\vec{q})$ is the static structure factor $S(\vec{q})$, while $\left\langle\mathscr{S}_{e}(0)\right\rangle$ is related to the constant-volume specific heat, $C_{V}$ as $T^{2} C_{V}=$ $N\left(\sigma_{0}\right)^{6}\left[\left\langle\mathscr{S}_{e}(0)\right\rangle-\langle e\rangle^{2}\right]$.

\section{THE LOCAL SWAP MONTE CARLO ALGORITHM}

We study a $50 \%$ mixture of particles interacting through the pair potential $V_{\alpha \beta}(r)=\varepsilon\left[\left(\sigma_{\alpha}+\sigma_{\beta}\right) / r\right]^{12}+C_{\alpha \beta}$, where $\alpha, \beta=A, B$, with a cutoff at $r_{\mathrm{c}}=\sqrt{3} \sigma_{0}\left(\sigma_{0}\right.$ is the unit length). The choice $\sigma_{B}=1.2 \sigma_{A}$ hampers crystallization. We impose $\left(2 \sigma_{A}\right)^{3}+2\left(\sigma_{A}+\sigma_{B}\right)^{3}+\left(2 \sigma_{B}\right)^{3}=$

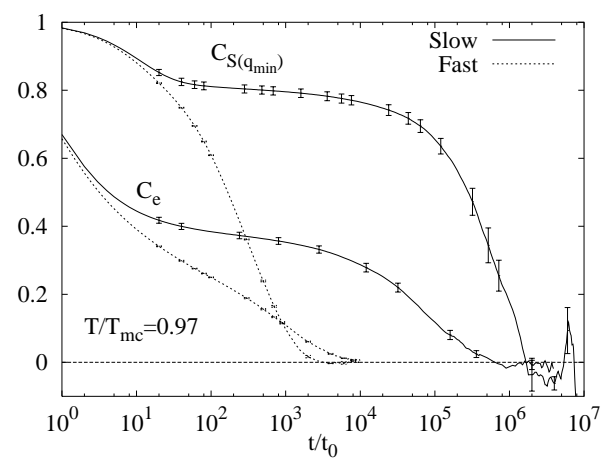

FIGURE 1. Correlators for two translationally invariant quantities: the potential energy density, $e$, and the square of the density fluctuation, $\mathscr{S}\left(q_{\mathrm{min}}\right)$, at the minimal momentum allowed by the boundary conditions (1024 particles, below $T_{\mathrm{mc}}$ ). The local swap algorithm decorrelates faster than standard MC.

$4 \sigma_{0}^{3}$. Constants $C_{\alpha \beta}$ are chosen to ensure continuity at $r_{\mathrm{c}}$. The simulations are at constant volume, with particle density fixed to $\sigma_{0}^{-3}$ and temperatures in the range $\left[0.897 T_{\mathrm{mc}}, 10.792 T_{\mathrm{mc}}\right]$, where $T_{\mathrm{mc}}$ is the Mode Coupling temperature [13]. We use periodic boundary conditions on a box of size $L$ (which discretizes momenta in units of $\left.q_{\min }=2 \pi / L\right)$ in systems with $N=512,1024,2048$ and 4096 particles. For argon parameters, $\sigma_{0}=3.4 \AA$, $\varepsilon / k_{B}=120 \mathrm{~K}$ and $T_{\mathrm{mc}}=26.4 \mathrm{~K}$.

We modify the Grigera-Parisi swap algorithm[14] to make it local, in order to keep the algorithm in the dynamic Universality Class [9] of standard Monte Carlo (MC). The elementary MC step is either (with probability $p$ ) a single-particle displacement attempt or (with probability $1-p$ ) an attempt to swap particles. The swap is made by picking a particle at random and trying to interchange its position with that of a particle of opposite type, chosen at random among those at distance smaller than $0.6 r_{\mathrm{c}}$. Detailed balance requires that the Metropolis test include not only the energy variation but the change in the number of neighbors. The swap acceptance is independent of system size and grows from $0.74 \%$ at $0.9 T_{\mathrm{mc}}$ up to $6 \%$ at $2 T_{\mathrm{mc}}$. In this work we use $p=0.5$ (named local swap from here on) and $p=1.0$ (named standard $\mathrm{MC})$. The time unit $t_{0}$ is $N / p$ elementary steps.

In fig. (1) we show that the time-correlator of the local swap is in general different from the one of the standard MC. The main difference is given by the absence of the cage effect when the particles are allowed to swap. This is seen by the absence of the plateau in the time correlator in the swap dynamics. This implies that Mode Coupling transition is rather ill-defined within the swap dynamics. In fact, at mean field level Mode Coupling singularity is seen as due to the divergence of the caging time scale [13]. The Theory of Critical Phenomena suggests that the decay of time correlators of two different 
TABLE 1. Stretching exponent $\beta$ of the time correlator of the energy at different temperatures. The fit to a stretched exponential form has been performed in the region $C<0.1$

\begin{tabular}{rrrrrrr}
\hline$T$ & $2.13 T_{\mathrm{mc}}$ & $1.08 T_{\mathrm{mc}}$ & $1.02 T_{\mathrm{mc}}$ & $0.97 T_{\mathrm{mc}}$ & $0.92 T_{\mathrm{mc}}$ & $0.89 T_{\mathrm{mc}}$ \\
\hline$\beta$ & $0.99(3)$ & $0.99(8)$ & $0.98(12)$ & $0.89(13)$ & $0.83(4)$ & $0.93(12)$ \\
\hline
\end{tabular}

dynamics sharing both the local nature and the conservation laws is given by the same fuction of the correlation length $\xi$ (up to non universal numerical prefactors). They are said in fact to belong to the same dynamic Universality Class [9]. Since at very long times that decay is expected to be exponential it is possible to define the exponential autocorrelation time [15] which identifies the longest characteristic relaxation time $\tau$ :

$$
C_{O}(t) \underset{t \rightarrow \infty}{\longrightarrow} \mathrm{e}^{-t / \tau}
$$

where

$$
\tau \sim \xi^{z}
$$

the dynamic exponent $z$ depending only the on the Class of the dynamics.

In a critical phenomenon one expects that eventually all the observables with the same symmetry decay with the same rate $1 / \tau$ since they are all coupled to the slowest mode. The order parameter is supposed to be such slowest mode and its decay is purely exponential. In fig. (2) we show the large time decay of different translational invariant quantities obtained with the local swap. At high temperatures the exponential time is clearly the same, on the other hand at lower temperatures this is more difficult to show, since the point where in log-linear representation all the curves become parallel moves at lower and lower values of $C$. Actually, in literature the time decay of glass-former is more often described by stretched exponentials like $C_{O}(t) \sim \exp -(t / \tau)^{\beta}$, with $\beta<1$. The stretching would arise from the contribution of many regions relaxing with different time scales. For the quantities we study in this work the stretched exponential fit nicely the time correlators only at intermediate times while, as we show in table 1 in the asymptotic region $C<0.1$ the decay is in fact purely exponential. It would be very difficult to get this result with standard algorithm since at large $t$ the correlator is very noisy.

The advantage of local swap over standard MC is not limited to the lack of the cage effect. This would be of no use if the exponential time of the two dynamics was the same. We see instead in Fig. (3) that, although the $\tau$ of the local swap seems to diverge, it remains always two order of magnitude smaller than the exponential time for the standard MC. Because of eq. (6) the fact that this ratio is roughly constant at all temperatures suggests that $z$ is the same for both dynamics and then that they belong to the

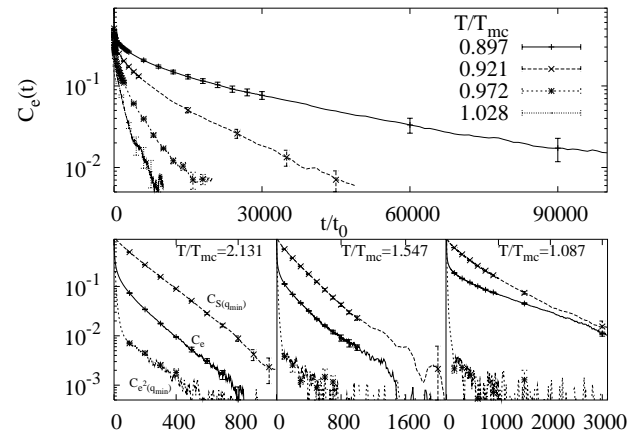

FIGURE 2. Time correlators for $N=1024$ particles $(N=$ 2048 at $T / T_{\mathrm{mc}}=0.897$ ) with the swap algorithm. Top panel: temperature variation of the correlator of $e$, close to $T_{\mathrm{mc}}$. Bottom panels: correlators of several translational invariant quantities. Asymptotically, three parallel straight lines should be found, with slope $-1 / \tau_{\exp }$. At the highest temperature, (bottom,left), this is clearly observed. At lower temperatures (bottom,right) we do not have enough independent measurements to see clearly this common slope.

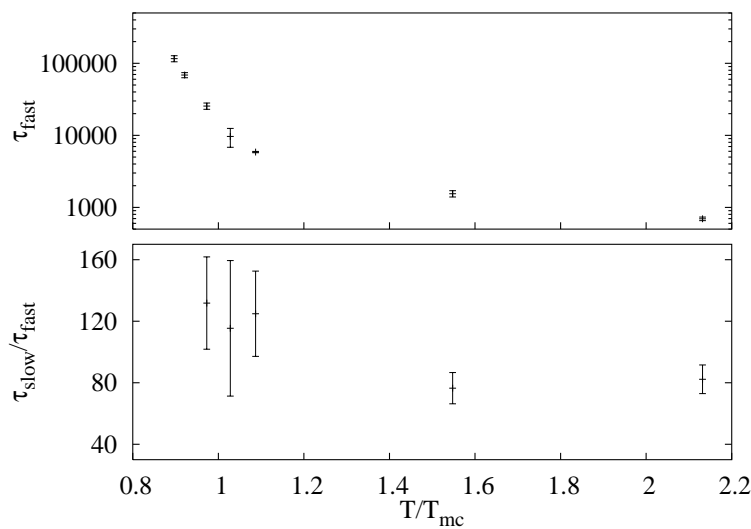

FIGURE 3. Top: exponential time $\tau_{\text {fast }}$ of the translational invariant quantities with the swap dynamics for $N=1024$ particles $\left(N=2048\right.$ at $\left.T / T_{\mathrm{mc}}=0.897\right)$. Bottom: the ratio of the exponential times for standard MC $\left(\tau_{\text {slow }}\right)$ and local swap dynamics $\left(\tau_{\text {fast }}\right)$.

same Universality class. As anticipated in the introduction, reducing $\tau$ allows us to obtain results with an higher statistical accuracy. We show in fact in table 2 some parameters describing a selection of recent numeric simulations using standard algorithms (molecular dynamics) 
sphere model [16] with $\sigma_{B}=1.4 \sigma_{A}$, the swap acceptance reduces of two order of magnitudes.

Finite size effects Having looked for a diverging correlation length we must at the moment content with a static correlation length that, close to the glass transition, is an order of magnitude larger than the range of the interactions. This challenges the common view that in glasses only dynamic quantities become correlated at large scales. More importantly, the study of the dynamics of translationally invariant quantities appears as a challenge to experimentalists. While measurements of the frequency dependence of the specific heat [16, 22] are an appealing possibility to estimate the potential energy relaxation time, the correlation-length could be studied by Finite-Size Scaling of the specific-heat and of relaxation times in films or in larger pores than previously used to confine glass-formers. In fact experiments in films [23] and nanopores 24] show that the glass transition changes in samples with one or more dimensions of nanometric scale ${ }^{2}$. Interestingly, the specific-heat of toluene confined on pores of diameter $8.7 \mathrm{~nm} \mathrm{[24],} \mathrm{close} \mathrm{to} \mathrm{its} \mathrm{glass} \mathrm{tempera-}$ ture, is significantly smaller than for bulk toluene, which could signal a correlation length well above the nanometric scale.

At a qualitative level one may note that a second order phase transition is not the only scenario predicting Finite Size effects in the specific heat. In fact, in the random first order transition picture of the glass transition [25] recently revised by Biroli and Bouchaud [26] the contribution to the specific heat from the configurational entropy $s_{c}(T)$ (the logarithm of the number of metastable states) is sizeable only when the system is large enough to contain many regions in different states. This happens when

$$
L^{d} s_{c}(t)>1 \text {. }
$$

To discriminate between the two interpretations a direct measurement of the correlation length of the energy would be needed. Nevertheless, in [27] we have shown some evidence for a singular behavior of the specific heat, consistent with the second order phase transition interpretation.

\section{ACKNOWLEDGMENTS}

We thank G. Biroli for pointing out that the random first order transition picture may explain finite size effects in

\footnotetext{
2 although it is difficult to disentangle Finite-Size Scaling from the effects of the interaction with the substrate.
}

the specific heat. P.V. was supported by the EC (contract MCFI-2002-01262). We were partly supported by MEC (Spain), through contracts BFM2003-08532, FIS200405073 and FPA2004-02602. The total CPU time devoted to the simulation (carried out at BIFI PC clusters) amounts to 10 years of $3 \mathrm{GHz}$ Pentium IV.

\section{REFERENCES}

1. L. Chayes, V. J. Emery, S. A. Kivelson, Z. Nussinov, and G. Tarjus, Physica A Statistical Mechanics and its Applications 225, 129-153 (1996).

2. C. Donati, S. Franz, G. Parisi, and S. C. Glotzer, J. Non-Crys.Sol. 307, 215-224 (2002).

3. H. E. Castillo, C. Chamon, L. F. Cugliandolo, J. L. Iguain, and M. P. Kennett, Phys. Rev. B 68, 134442-1-134442-41 (2003).

4. G. Biroli, and J.-P. Bouchaud, Europhys. Lett. 67, 21-27 (2004).

5. S. Whitelam, L. Berthier, and J. P. Garrahan, Phys. Rev. Lett. 92, 185705-1-185705-4 (2004).

6. P. G. Debenedetti, Metastable Liquids, Princeton University Press, 1997.

7. C. A. Angell, K. L. Ngai, G. B. McKenna, P. F. McMillian, and S. W. Martín, J. Appl. Phys. 88, 3133-3156 (2000).

8. P. G. Debenedetti, and F. H. Stillinger, Nature 410, 259-267 (2001).

9. J. Z. Justin, Quantum Field Theory and Critical Phenomena, Oxford University Press, 2002.

10. J. Cardy, Scaling and Renormalization in Statistical Physics, Cambridge University Press, Cambridge, 1996.

11. D. Amit, and V. Martín-Mayor, Field Theory, the Renormalization Group and Critical Phenomena, World Scientific Singapore, in press, 2005.

12. L. Berthier, Phys. Rev. Lett. 91, 055701-1-055701-4 (2003).

13. W. Götze, and L. Sjögren, Rep. Prog. Phys. 55, 241-336 (1992).

14. T. S. Grigera, and G. Parisi, Phys. Rev. E 63, 045102-1045102-4 (2001).

15. A. D. Sokal, in Functional Integration: Basics and Applications, eds. C. DeWitt-Morette, P. Cartier and A. Folacci, Plenum, New York, 1997.

16. C. C. Yu, and H. M. Carruzzo, Phys. Rev. E 69, 051201-1-051201-10 (2004).

17. L. Berthier, Phys. Rev. E 69, 020201-1-020201-4 (2004).

18. N. Lacevic, F. W. Starr, T. B. SchrÃÿder, and S. C. Glotzer, J. Chem. Phys. 119, 7372-7387 (2003).

19. K. Kim, and R. Yamamoto, Phys. Rev. E 61, R41-R44 (2000).

20. M. D. Ediger, Annu. Rev. Phys. Chem. 51, 99-128 (2000).

21. E. V. Russell, and N. E. Israeloff, Nature 408, 695-698 (2000).

22. N. O. Birge, and S. R. Nagel, Phys. Rev. Lett. 54, 2674-2677 (1985).

23. D. S. Fryer, et al., Macromolecules 34, 5627 (2001).

24. D. Morineau, Y. Xia, and C. Alba-Simionesco, J. Chem. Phys. 117, 8966-8972 (2002).

25. T. Kirkpatrick, D. Thirumalai, and P. Wolynes, Phys. Rev. A 40, 1045-1054 (1989). 
26. G. Biroli, and J.-P. Bouchaud, J. Chem. Phys. 121, 7347-7354 (2004)

27. L. A. Fernandez, V. Martin-Mayor, and P. Verrocchio, preprint condmat $\mathbf{0 5 0 4 3 2 7}$ (2005). 